\title{
Are the Anomalies in Water Due to an Unapproachable Critical Point?
}

Thermal conductivity of supercooled water

Authors:John W. Biddle, Vincent Holten, Jan V. Sengers, and Mikhail A. Anisimov. arXiv:1302.6280

Phys. Rev. E 87, 042302 (2013)

Recommended with a commentary by Marivi Fernandez-Serra, Stony Brook University

Water in both its liquid and solid forms is a fascinating system. New "anomalies" to add to their long list of anomalies are still being discovered. There are so many of them that trying to find a common link between them might appear futile. However, a large number of experimental and theoretical works in the last few years have focussed on a particular side of the story that may serve as the basis for a unifying theory to explain most of the liquid anomalies both at high and low temperatures. The basic idea is that they might all originate from the existence of a second critical point, hidden somewhere in the supercooled region of the phase diagram of liquid water.

Supercooled water is a metastable phase in which water remains in a liquid form below the melting temperature $\left(\mathrm{T}_{M}=273 \mathrm{~K}\right.$ at atmospheric pressure) and above the homogeneous nucleation temperature $\left(\mathrm{T}_{H} \approx 231 \mathrm{~K}\right)$. The thermodynamic response functions of water (isothermal compressibility, isobaric heat capacity and thermal expansivity) in this region seem to diverge when the temperature approaches a critical value $\mathrm{T}_{C}$ [ J. Phys. Chem. 77, 3092 (1973)] as in a standard critical behavior. The problem is that $\mathrm{T}_{C}$ is approximately 228 $\mathrm{K}$, a temperature below $\mathrm{T}_{H}$, and therefore not accessible to experiments. So the burning question is: does this second "virtual" critical point really exist, and even more importantly, if it exists, what are the two phases separated by a line of first order phase transitions that coelesce at the critical point? A short answer to the second question is that, the polymorphism of the crystalline phases of ice (there are 15 known different crystalline ice structures and more than three amorphous structures) may occur also in water. Indeed, it is suggested that in the low $\mathrm{T}$ high $\mathrm{P}$ region of supercooled water two different liquids, a low density liquid (LDL) and a high density liquid (HDL) are separated by a coexistence line that ends at the aforementioned critical point. As for the first question, there is no short answer, the yes and no are still being heavily debated.

What makes the highlighted paper really interesting and different from many others studying the supercooled region of the phase diagram of water is not so much the measurement of the thermal conductivity itself but the similarities and differences drawn between the two critical points of liquid water. The vapor-liquid critical point of liquid water is a text book example of critical behavior, belonging to the universality class of the threedimensional Ising model. In the vicinity of this point the correlation length of the density fluctuations in liquid water diverges. As a consequence, its transport properties like viscosity or thermal conductivity are enhanced. This enhancement is characterized by a singular behavior that can be expressed analytically as powers of the correlation length with universal critical exponents.

The thermal conductivity is directly proportional to the density of the fluid $\rho$, its specific heat capacity $\mathrm{C}_{p}$ and its thermal diffusivity $\mathrm{D}_{t}$. Near the critical point, the $\mathrm{D}_{t} \rightarrow 0$ 
according to the Stokes-Einstein relation ( in which $\mathrm{D}_{t}$ is proportional to $\mathrm{T}$ and inversely proportional to both the viscosity and the particle radii), with the particle size being replaced by the correlation length. However, despite this, the thermal conductivity diverges near the critical point, because the divergence of $\mathrm{C}_{p}$ is stronger than the divergence of the correlation length. For the vapor-liquid critical point region of the phase diagram, the critical enhancement of the the thermal conductivity is felt far away from the critical point, primarily due to the dominance of the Stokes-Einstein law for the thermal diffusivity in this region. If the same situation would prevail for the liquid-liquid critical point, one would expect the measured thermal conductivity of supercooled water would strongly increase in the accessible region away from the critical point. The authors prove that this is not the case, highlighting important differences between the two critical points. First of all, the thermal conductivity of supercooled water has been measured only down to $250 \mathrm{~K}$ [ Phys. Rev. E 74, 031502 (2006)] (still quite an impressive achievement, taking into account that this is a metastable liquid phase). The thermal conductivity decreases with temperature and shows no anomalous behavior whatsoever. The authors show that this may not be incompatible with the existence of the liquid-liquid critical point. The viscosity of supercooled water increases dramatically in supercooled water, much more than in the vicinity of the vaporliquid critical point. As explained above, the thermal conductivity is inversely proportional to the viscosity. Therefore, despite the divergent behavior of the specific heat capacity, the viscosity enhancement largely suppresses the thermal conductivity enhancement. The theoretical model presented by the authors predicts that indeed there should be a critical effect but only very close to the critical temperature, and completely undetectable far from it.

The authors also note a very important difference between the two critical points. While in the liquid-vapor critical point fluctuations in the density are dominated by the diffusive behavior of the molecules, near the liquid-liquid critical point density fluctuations are governed by both such diffusive behavior and the structural relaxations between two very different liquid structures, were they to exist. Differences in the transport properties of the liquid and in the temperature dependence of the order parameter may be expected to be extracted in future experiments and theoretical investigations, especially those related to sound propagation.

In conclusion, the approach of investigating the similarities and differences between the two critical points (one real and one only predicted) in liquid water is a fresh departure from the studies focused only on the supercooled region. Taking into account that some of the liquid anomalies such as the isothermal compressibility minimum occur at rather high temperatures $(\sim 320 \mathrm{~K})$, any model predicting the existence of this second critical point, should also explain, predict and relate it to the thermodynamic anomalies in the high temperature region of the liquid phase diagram. 